\title{
Anthranoid laxative use is not a risk factor for colorectal neoplasia: results of a prospective case control study
}

\author{
G Nusko, B Schneider, I Schneider, Ch Wittekind, E G Hahn
}

\begin{abstract}
Background-Anthranoid laxatives are the most commonly used purgatives in the therapy of acute and chronic constipation. Recent experimental data and a prospective cohort study provide evidence of a possible risk of anthranoid use for the development of colorectal neoplasms.

Materials and methods-We performed a prospective case control study at the University of Erlangen to investigate the risk of anthranoid laxative use for the development of colorectal adenomas or carcinomas. A total of 202 patients with newly diagnosed colorectal carcinomas, 114 patients with adenomatous polyps, and 238 patients (controls) with no colorectal neoplasms who had been referred for total colonoscopy were studied. The use of anthranoid preparations was assessed by standardised interview, and endoscopically visible or microscopic melanosis coli was studied by histopathological examination.
\end{abstract}

Results-There was no statistically significant risk of anthranoid use for the development of colorectal adenomas (unadjusted odds ratio $1.0 ; 95 \%$ CI $0.5-1.9$ ) or carcinomas (unadjusted odds ratio 1.0; 95\% CI 0.6-1.8). Even after adjustment for the risk factors age, sex, and blood in the stools by logistic regression analysis the odds ratio for adenomas was 0.84 (95\% CI $0.4-1.7)$ and for carcinomas 0.93 (95\% CI 0.5-1.7). Also, there were no differences between the patient and control groups for duration of intake. Macroscopic and high grade microscopic melanosis coli were not significant risk factors for the development of adenomas or carcinomas.

Conclusion-Neither anthranoid laxative use, even in the long term, nor macroscopic or marked microscopic melanosis coli were associated with any significant risk for the development of colorectal adenoma or carcinoma.

(Gut 2000;46:651-655)

Keywords: anthranoid laxatives, melanosis coli; colorectal adenoma; colorectal carcinoma

Laxatives containing a variety of different pharmaceutical ingredients are often used to treat acute and chronic constipation. They frequently contain osmotic saline or sugar solutions, anthranoids (senna, aloe), diphenylmethane derivates, or synthetic prokinetics. $^{1}$
Undesirable side effects such as abdominal pain, atonic colon, hypokalaemia, and pigmentation of the colonic mucosa (melanosis coli) have been described. ${ }^{1-3}$ Chronic ingestion of cathartics in the anthranoid group, such as cascara, senna, sagrada, frangula, aloes, or rhubarb is believed to be an aetiological factor in melanosis coli, and this has also been demonstrated experimentally. ${ }^{4}$ The term melanosis coli refers to the brownish black pigmentation of the colorectum. The composition of the pigment is essentially unknown. A recent histochemical study categorised it as a melanised ceroid and found a significant increase in apoptotic bodies in the colonic epithelium lining, indicating that this type of cell death is not due to natural programmed cell renewal but to the action of laxatives. ${ }^{5}$ Melanosis coli has long been considered to be a harmless discolouration of the large bowel. However, mutagenic effects have recently been reported for some anthranoid laxatives in vitro. ${ }^{67}$ Some evidence has been found in animal experiments that dantrone, a synthetic anthranoid laxative, may induce colorectal tumours. ${ }^{8}$ A recent study involving long term administration of purified senna extract to rats revealed no association with gastrointestinal, hepatic, renal, or adrenal tumours. $^{9}$

Evidence of a tumorigenic potential of anthranoid laxatives found in clinicalepidemiological studies is controversial. Several cohort studies failed to find any association between anthranoid laxative use or melanosis coli and colorectal cancer. ${ }^{10-13}$ Only a single case report ${ }^{14}$ and a recent prospective study ${ }^{15}$ have shown a higher risk for developing colorectal cancer in patients with melanosis coli and anthranoid laxative use. Experimental data indicate that melanosis coli is induced by anthranoid intake. However, $73.4 \%$ of patients with melanosis coli reported using anthranoid laxatives while $26.6 \%$ did not. The aim of our prospective case control study was to investigate the risk of colorectal neoplasia, not only in patients with melanosis coli, but also in patients reporting anthranoid laxative use.

Material and methods

We studied the risk of melanosis coli and laxative use for colorectal neoplasias in a prospective case control study carried out at the University of Erlangen, Germany (Departments of Medicine I, Surgery, and Pathology). Between 1993 and 1996 all consecutive patients who underwent total colonoscopy at the endoscopic units of the Department of 
Medicine I and Department of Surgery, and those who were referred for surgical resection of a known colorectal carcinoma were considered for participation. In accordance with the colorectal adenoma-carcinoma sequence, we defined two groups of patients comprising those suffering from a newly diagnosed colorectal carcinoma (carcinoma group) or colorectal adenoma only (adenoma group). Patients with a carcinoma and synchronous adenomas were placed in the carcinoma group. Patients with no colorectal neoplasias were used as controls. A sample size of 200 patients for each group was intended, on the assumption of a rate of $15 \%$ of laxative users in the control group. With such a sample size a significant difference between the controls and case groups at the $5 \%$ level can be expected, with a power of $80 \%$ for an odds ratio of 2.0 . Recruitment of study participants was stopped when more than 200 carcinoma patients had been enrolled.

Excluded from the study were all patients younger than 40 years and those who had suffered precancerous conditions or lesions (for example, a history of previous carcinoma, colorectal adenomas, FAP or the Lynch syndrome, cholecystectomy, ureterosigmoidostomy, radiation therapy, inflammatory bowel disease, or chronic diarrhoea). All patients and controls underwent total colonoscopy, with the exception of patients with a stenosing distal colorectal carcinoma. All endoscopic examinations were documented using standardised forms. To obtain evidence of microscopic melanosis coli, a biopsy was taken from the rectal and caecal mucosa of all patients and controls. All biopsies and endoscopic or surgical specimens of adenomas and carcinomas were examined histopathologically at the Department of Pathology, University of Erlangen, by one of the authors (ChW), who was blinded to the clinical data relating to laxative use, and were classified in accordance with the WHO classification. ${ }^{16}$ Microscopic melanosis coli was categorised as low or high grade depending on the extent of pigmentation as a result of accumulation of pigmented phagosomes of macrophages in the lamina propria. Laxative use, family history of carcinoma, dietary habits, clinical symptoms, and previous and current diseases were investigated using a standardised interview of all study participants on the day after colonoscopy. The interviewer was not blinded to the colonoscopic findings. All subjects were interviewed on their use not only of laxatives but also of any other anthranoid containing preparations using a checklist of substances compiled from a commonly used German pharmaceutical index (Rote Liste). This was necessary as a number of liver drugs and digestives also contain anthranoids. In the same interview the constituents of any medications taken, together with the frequency and duration of their use were recorded. Duration of use was calculated as an integral over lifetime.

Statistical analysis was performed by the Institute of Biometrics at the Medical School of Hannover, Germany, using the $\chi^{2}$ test and logistic linear regression. ${ }^{17}$ This analysis included patient age and duration of laxative use as continuous and all other factors as categorial variables (present $v$ absent).

The study was approved, unmodified, by the ethics committee.

\section{Results}

A total of 562 patients attending the medical and surgical departments of the University of Erlangen were considered for inclusion in the study. Eight patients were excluded: these had only hyperplastic polyps, and one patient had other non-neoplastic polyps. The final study population comprised 554 subjects, 263 females and 291 males; 202 patients had colorectal carcinomas, 114 colorectal adenomas only, while 238 patients with no colorectal neoplasia formed the control group.

The mean age of the study population was 58.47 years. Subjects in the control were, on average, younger ( 53.9 years) than those in the carcinoma (61.88 years) and adenoma (61.99 years) groups. In the adenoma and carcinoma groups there was a significant predominance of males ( $57 \%$ and $62 \%$, respectively) $(\mathrm{p}=0.001)$ while in the control group females $(58 \%)$ predominated. Thus age and male sex were significant risk factors for adenomas and carcinomas (table 1).

Blood in the stools was reported significantly more often by patients in the adenoma $(50 \%)$ and carcinoma (64\%) groups compared with controls $(41 \%)$. Smoking was significantly

Table 1 Risk factors and unadjusted odds ratios for adenomas and carcinomas (controls $n=238$; adenomas $n=114$; carcinomas $n=202$ )

\begin{tabular}{|c|c|c|c|c|c|}
\hline \multirow[b]{2}{*}{ Risk factor } & \multirow{2}{*}{$\begin{array}{l}\text { Controls } \\
(n(\%))\end{array}$} & \multicolumn{2}{|l|}{ Adenomas } & \multicolumn{2}{|c|}{ Carcinomas } \\
\hline & & $(n(\%))$ & OR $(95 \% C I)$ & $(n(\%))$ & OR $(95 \% C I)$ \\
\hline Age $\geqslant 65$ years & $73(31)$ & $65(57)$ & $3.0(1.9-4.8)$ & $119(59)$ & $3.2(2.2-4.8)$ \\
\hline Male sex & $100(42)$ & $65(57)$ & $1.8(1.2-2.9)$ & $126(62)$ & $2.3(1.6-3.4)$ \\
\hline CRC family history & $22(9)$ & $10(9)$ & $0.9(0.4-1.2)$ & $23(11)$ & $1.3(0.7-2.3)$ \\
\hline Abdominal pain & $170(72)$ & $73(64)$ & $0.7(0.4-1.1)$ & $95(48)$ & $0.4(0.2-0.5)$ \\
\hline Blood in stools & $86(36)$ & $57(50)$ & $1.8(1.1-2.8)$ & $130(64)$ & $3.2(2.2-4.7)$ \\
\hline Constipation & $5(2.1)$ & $2(1.8)$ & $0.8(0.2-4.3)$ & $9(4.5)$ & $2.2(0.7-6.6)$ \\
\hline Smoking & $97(41)$ & $66(58)$ & $2.0(1.3-3.1)$ & $101(50)$ & $1.5(1.0-2.1)$ \\
\hline Alcohol & $208(87)$ & $102(90)$ & $1.2(0.6-2.5)$ & $180(89)$ & $1.2(0.7-2.1)$ \\
\hline Fat diet & $28(12)$ & $22(19)$ & $1.8(0.9-3.3)$ & $33(16)$ & $1.5(0.8-2.5)$ \\
\hline Anthranoid laxative use & $33(14)$ & $16(14)$ & $1.0(0.5-1.9)$ & $29(14)$ & $1.0(0.6-1.8)$ \\
\hline Other laxative use & $8(3.4)$ & $4(3.5)$ & $1.0(0.3-3.5)$ & 7 (3.5) & $1.0(0.4-2.9)$ \\
\hline Endoscopic melanosis coli & $7(2.9)$ & $5(4.4)$ & $1.5(0.5-4.9)$ & $8(4.0)$ & $1.4(0.5-3.8)$ \\
\hline Microscopic melanosis coli & $28(12)$ & $23(20)$ & $1.9(1.0-3.5)$ & $48(24)$ & $2.3(1.4-3.9)$ \\
\hline Low grade microscopic melanosis coli & $16(7)$ & $16(14)$ & $2.3(1.1-4.7)$ & $38(19)$ & $3.2(1.7-6.0)$ \\
\hline High grade microscopic melanosis coli & $12(5)$ & $7(6)$ & $1.2(0.5-3.2)$ & $10(5)$ & $1.0(0.4-2.3)$ \\
\hline
\end{tabular}


more common among patients with adenomas $(58 \%)$ and carcinomas (50\%) than among controls $(41 \%)$. Abdominal pain was present significantly less frequently in the carcinoma $(48 \%)$ than in the adenoma patients $(64 \%)$ or controls $(72 \%)$. A family history of colorectal carcinomas, constipation, alcohol consumption, and dietary habits (high fat intake) showed no significant association with the presence of adenomas or carcinomas.

\section{ANTHRANOID LAXATIVE USE AND COLORECTAL} NEOPLASMS

A total of $97(17.5 \%)$ study participants (cases and controls) admitted to using laxatives. Anthranoid containing laxatives were used by $78(14.1 \%)$ participants and were thus the most common purgatives taken. They were used significantly $(\mathrm{p}<0.01)$ more often by females $(20.2 \%)$ than males $(8.6 \%)$. There was no significant difference in the use of anthranoid laxatives between adenoma patients and controls or carcinoma patients and controls (table 1). This indicates that the use of anthranoid laxatives is not a significant risk factor for colorectal neoplasms.

There was no statistically significant risk of anthranoid use for the development of colorectal adenomas (unadjusted odds ratio 1.0; 95\% CI 0.5-1.9) or carcinomas (unadjusted odds ratio $1.0 ; 95 \%$ CI $0.6-1.8$ ). There were significant differences in age, sex, and some anamnestic findings (table 1) between cases and controls, and the odds ratio was therefore adjusted for the risk factors age, sex, and blood in the stools by logistic regression. The adjusted odds ratio of anthranoid laxative use for adenoma patients was 0.84 (95\% CI $0.41-$ 1.71 ) and for carcinoma patients $0.93(95 \%$ CI 0.51-1.70); the adjusted odds ratio also failed to show any significantly increased risk of adenoma or carcinoma by anthranoid laxative use. The mean duration of anthranoid laxative use was 14.8 years in the control, 12.6 years in the adenoma, and 12.9 years in the carcinoma groups. Duration of anthranoid laxative use was included in the logistic regression as a continuous variable and showed no significant influence on the development of adenoma or carcinoma $(p=0.25$ and 0.41 , respectively).

ANTHRANOID LAXATIVE USE AND MELANOSIS COLI Anthranoid laxative use was significantly associated with macroscopic and microscopic melanosis coli $(\mathrm{p}<0.01)$. Nine $(12 \%)$ patients using anthranoid laxatives showed macroscopic and $31(40 \%)$ microscopic melanosis coli. Eleven $(2 \%)$ of 476 non-users of such laxatives showed macroscopic, and 68 (14\%) microscopic melanosis (table 2). Low grade

Table 2 Association between melanosis coli and use of anthranoid laxatives

\begin{tabular}{lccl}
\hline & \multicolumn{2}{l}{ Anthranoid laxative use } \\
\cline { 2 - 4 } & Yes $(n=78)$ & No $(n=476)$ & Odds ratio (95\% CI) \\
\hline Endoscopic melanosis coli & $9(12 \%)$ & $11(2 \%)$ & $5.5(2.2-13.7)$ \\
Microscopic melanosis coli & $31(40 \%)$ & $68(14 \%)$ & $4.0(2.4-6.7)$ \\
Low grade microscopic melanosis coli & $17(22 \%)$ & $53(11 \%)$ & $2.2(1.2-4.1)$ \\
High grade microscopic melanosis coli & $14(18 \%)$ & $15(3 \%)$ & $6.7(3.1-14.6)$ \\
\hline
\end{tabular}

microscopic melanosis coli was found in $22 \%$ of anthranoid laxative users and in $11 \%$ of non-users, while high grade microscopic melanosis was found in $18 \%$ of users and in 3\% of non-users. The association between melanosis coli and anthranoid laxative use was much stronger for high grade than for low grade microscopic melanosis coli (table 2).

No significant association was found between endoscopically evident (macroscopic) melanosis coli or high grade microscopic melanosis coli and colorectal neoplasms (table 1). Low grade microscopic melanosis coli was significantly associated with carcinomas but not with adenomas (table 1 ).

\section{Discussion}

Colorectal cancer is a major cause of tumour related deaths in the western world. ${ }^{18}$ While most colorectal cancers are believed to be caused by environmental factors, ${ }^{19-21}$ dietary habits appear to be involved in the increased prevalence of colorectal cancer in the industrial nations. ${ }^{12} 22$ Recent experimental data and a prospective cohort study appear to provide evidence of a possible risk of anthranoid laxative use for the development of colorectal neoplasms. ${ }^{6715}$ Constipation and the use of laxatives have been reported in $15-20 \%$ of the US population. ${ }^{23} 24$

In our study, laxative use was admitted by $17.5 \%$ of all subjects in a standardised interview. Anthranoids were the most common constituents $(80.4 \%)$ of the laxatives and were used to a comparable extent by both controls and patients with colorectal adenomas or carcinomas. There were no differences between these two groups in terms of frequency and duration of anthranoid use. Even long term use over more than 20 years was not associated with any significantly higher risk of developing colorectal neoplasias.

The association of bowel habits and laxative use with colorectal cancer was examined in the Melbourne Colorectal Cancer Study. ${ }^{11}{ }^{12}$ This study demonstrated that previous use of anthranoid laxatives and phenolphthalein containing laxatives was not associated with any increased risk of colorectal cancer. However, a methodological shortcoming of this study was the fact that the control subjects were assessed on the basis of a personal interview only. As the control subjects were not examined by total colonoscopy, as in our study, possible asymptomatic colorectal adenomas or carcinomas in the control group cannot be ruled out.

A meta-analysis of 14 previously published case control studies ${ }^{25}$ revealed statistically significant risks of colorectal cancer associated with both constipation and the use of cathartics. This risk, however, appeared to reflect the confounding influence of dietary habits rather than the primary influence of bowel habits and laxative use, as both constipation and the use of laxatives were associated with much lower odds ratios than various dietary constituents, such as fat, meat, alcohol, and low vegetable or low residue diets. ${ }^{125}$

The reported prevalence of endoscopically visible melanosis coli in proctoscopic studies 
varies between $0.8 \%$ and $9.3 \% .{ }^{26}{ }^{27}$ In constipated patients, macroscopic melanosis coli has been observed in $12-31 \%$ of cases. ${ }^{28}$ In the present study we found macroscopic melanosis coli in $3.6 \%$ of all participants. There were no significant intergroup differences in terms of the prevalence of macroscopic melanosis coli. Previous proctoscopic analyses reported a comparable association of colorectal carcinoma and melanosis coli of $4-5 \% .{ }^{26}{ }^{27} \mathrm{~A}$ significant association of melanosis coli and colorectal cancer $(18.6 \%)$ has recently been reported in a prospective colonoscopic cohort analysis. ${ }^{15}$ In that study melanosis coli was assumed to be a reliable marker of chronic anthranoid laxative abuse. Unfortunately, the use of laxatives was only confirmed for the 33 patients with colorectal adenomas or carcinomas, but not for the other participants in the study. Also, the group of patients with carcinoma comprised 11 individuals only, and was thus too small to permit the conclusion that there is a significant association of melanosis coli and colorectal cancer. If melanosis coli were a risk factor for developing colorectal cancer, colorectal adenomas should also be significantly associated with melanosis coli, in accordance with the concept of the colorectal adenoma-carcinoma sequence, ${ }^{29-33}$ but this was not the case in the above mentioned study.

For melanosis coli to be considered a precancerous condition of colorectal carcinoma, it would have to be demonstrable for at least 10 years prior to the carcinoma, and colorectal adenomas would also have to be significantly associated with melanosis coli. In a retrospective cohort study ${ }^{13}$ a significant association of colorectal adenomas, but not of colorectal carcinomas, with macroscopic melanosis coli was found. However, the adenomas associated with melanosis coli were significantly smaller than those without such an association, and were found predominantly in the proximal colonic segments in which pigmentation is usually most marked. ${ }^{34}$ Adenomas located in these bowel segments are easier to detect as they do not contain the pigment. ${ }^{35}$ Only in one case has an adenomatous polyp been reported to contain the melanotic pigment. ${ }^{36}$ In this study, ${ }^{13}$ adenomas associated with melanosis coli did not harbour a greater amount of villous structure, nor did they contain high grade dysplasia more often than adenomas not surrounded by melanosis. Small and tubular adenomas are not likely to harbour severe dysplasia or invasive carcinoma. ${ }^{30}{ }^{37-44}$ The significant association of colorectal adenomas with melanosis coli, found retrospectively, might be explained by the ease with which they are detected endoscopically. The question of whether melanosis coli might merely lead to the development of only small adenomas which do not progress to invasive carcinomas needs to be studied by molecular analysis of oncogene or tumour suppressor gene mutations. ${ }^{45-48}$

Microscopic melanosis is found more often than endoscopically visible melanosis coli. In an unselected autopsy series, microscopic melanosis was found in $59.5 \%$ of cases. ${ }^{34} \mathrm{~A}$ histopathological study revealed that among 45 patients with microscopic melanosis coli, the pigmentation was macroscopically visible in only 14 cases $(31 \%) .{ }^{4}$ Laxatives may be associated with, but need not cause, microscopic melanosis. Also, the luminal bacterial flora and faecal material may have a toxic effect on the surface epithelium. ${ }^{34}$ For all cases and controls in our study, biopsy specimens were histopathologically scrutinised for microscopic melanosis coli which was categorised as low or high grade pigmentation. High grade microscopic melanosis was significantly associated with anthranoid laxative use but not with colorectal adenomas or carcinomas. Low grade microscopic melanosis was found to be significantly associated with colorectal carcinoma but not with adenomas. We suspect that this might be due to a histopathological mucosal reaction to coprostasis in carcinomas.

There are good reasons for not considering melanosis coli as a reliable marker of chronic anthranoid laxative use as only $73.4 \%$ of patients with melanosis coli admitted to anthranoid use. ${ }^{4}$ Furthermore, anthranoids are also found in some preparations of liver drugs, digestives, or alternative herbal remedies. ${ }^{49}$ Experimental data have shown that at least four months' consumption of anthranoid containing laxatives is necessary for the development of macroscopic melanosis coli. In withdrawal tests the pigmentation was reversible within 5-15 months in a large proportion of patients. ${ }^{40}{ }^{51}$ In our study, anthranoid laxative use was significantly associated with melanosis coli. But melanosis coli was found in only about $40 \%$ of these patients. This might be explained by the fact that melanosis coli had already disappeared by the time of examination as $27.5 \%$ of patients using anthranoid containing preparations reported an interval of more than one year since the last use.

In conclusion, macroscopic melanosis coli should not be assumed to be either a reliable marker of chronic anthranoid laxative use or a precancerous condition. Nor is there any evidence of a significant risk of even long term anthranoid laxative use for the development of colorectal neoplasms.

1 Müller-Lissner S. Nebenwirkungen von Laxantien. $Z$ Gastroenterol 1992;30:418-27.

2 Leng-Peschlow E. Senna and its rational use. Pharmacology 1992;44(suppl 1):1-52.

3 Müller-Lissner SA. Adverse effects of laxatives: fact and fiction. Pharmacology 1993;47(suppl 1):138-45.

4 Badiali D, Marcheggiano A, Pallone F, et al. Melanosis of the rectum in patients with chronic constipation. Dis Colon Rectum 1985;28:241-5.

5 Benavides SH, Morgante PE, Monserrat AJ, et al. The pigment of melanosis coli: a lectin histochemical study. Gastrointest Endosc 1997; 46 : 131-8.

6 Westendorf J, Marquardt H, Poginsky B, et al. Genotoxicity of naturally occurring hydroxyanthraquinones. Mutation Res 1990;240:1-12.

7 Tikkanen L, Matsushima T, Natori S. Mutagenicity of anthraquinones in the Salmonella pre-incubation test. Mutation Res 1983;116:297-304.

8 Mori H, Sugie S, Niwa K, et al. Induction of intestinal tumours in rats by chrysazine. Br f Cancer 1985;52:781-3.

9 Lyden-Sokolowski A, Nilsson A, Sjöberg P. Two-year carcinogenicity study with sennosides in the rat: emphasis on gastro-intestinal alterations. Pharmacology 1993;47(suppl 1):209-15.

10 Gardiner JS, Walker SA, Macleari AJ. A retrospective mortality study of substituted anthraquinone on dye stuff tality study of substituted anthraquinon
workers. Br f Indust Med 1982;39:355-60.

11 Kune GA. Laxative use not a risk for colorectal cancer: data from the Melbourne Colorectal Cancer Study. $Z$ Gastroenterol 1993;31:140-3. 
12 Kune GA, Kune S, Field B, et al. The role of chronic constipation, diarrhea, and laxative use in the etiology of Cancer Study. Dis Colon Rectum 1988;31:507-12.

13 Nusko G, Schneider B, Ernst H, et al. Melanosis coli-a harmless pigmentation or a precancerous condition? $Z$ Gastroenterol 1997;35:313-18.

14 Patel PM, Selby PJ, Deacon J, et al. Anthraquinone laxatives and human cancer: An association in one case. Postgrad Med f 1989;65:216-17.

15 Siegers C-P, Hertzberg-Lottin Evon, Otte M, et al. Anthranoid laxative abuse - a risk for colorectal cancer? Gut 1993;34:1099-101.

16 Jass JR, Sobin LH. Histological typing of intestinal tumours. WHO international classification of tumours, 2 nd edn. Berlin: Springer, 1989

17 Breslow NE, Day NE. Statistical methods in cancer research. Vol. I-The analysis of case control studies. Lyon: IARC, 1980

18 Parkin DM, Läärä E, Muir CS. Estimation of worldwide frequency of sixteen major cancers in 1980. Int $\mathcal{F}$ Cancer frequency of sixteen

19 Thun MJ, Calle EE, Namoodiri MM, et al. Risk factors for fatal colon cancer in a large prospective study. 7 Natl Cancer Inst 1992;84:1491-500.

20 Boeing H, Wahrendorf J. Epidemiologie kolorektaler Karzinome. Internist 1991;32:306-14.

21 Devesa SS, Chow WH. Variation in colorectal cancer incidence in the United States by subsite of origin. Cancer 1993;71:3819-26.

22 Birt DF. The influence of dietary fat on carcinogenesis: lessons from experimental models. Nutr Rev 1990;48:1-5.

23 Everhart JE, Go VLW, Johannes S, et al. A longitudinal survey of self-reported bowel habits in the United States. Dig Dis Sci 1989:34:1153-62.

24 Dent OF, Goulston KJ, Zubrzycki J, et al. Bowel symptoms in an apparently well population. Dis Colon Rectum 1986;29:243-7.

25 Sonnenberg A, Müller AD. Constipation and cathartics as risk factors of colorectal cancer: a meta-analysis. Pharmarisk factors of colorectal cancer:
cology 1993;47(suppl 1):224-33.

26 Wittoesch JH, Jackman RJ, McDonald JR. Melanosis coli: general review and study of 887 cases. Dis Colon Rectum 1958;1:172-80.

27 Göbel D. Melanosis coli. Med Klin 1978;73:519-23.

28 Bockus HL, Williard JH, Bank J. Melanosis coli. The etiologic significance of the anthracene laxatives. A report of forty-one cases. $\mathcal{F} A M A 1933 ; 101: 1-6$.

29 Hermanek P. Dysplasie-Karzinom-Sequenz im Kolorektum. Zentralbl Chir 1992;117:476-82.

30 Hermanek P, Frühmorgen P, Guggenmoos-Holzmann I, et al. The malignant potential of colorectal polyps-A new statistical approach. Endoscopy 1983;15:16-20.

31 Matek W, Hermanek P, Demling L. Is the adenomacarcinoma sequence contradicted by the differing location of colorectal adenomas and carcinomas? Endoscopy 1986; 18:17-19.

32 Muto T, Bussey HJR, Morson BC. The evolution of cancer of the colon and rectum. Cancer 1975;36:2251-70.
33 Thierney RP, Ballantyne GH, Modlin IM. The adenoma to carcinoma sequence. Surg Gynecol Obstet 1990;171:81-94. 34 Koskela E, Kulju T, Collan Y. Melanosis coli. Prevalence, distribution, and histologic features in 200 consecutive autopsies at Kuopio University Central Hospital. Dis Colon Rectum 1989;32:235-9.

35 Morgenstern L, Shemen L, Allen W, et al. Melanosis coli. Changes in appearance when associated with colonic neoplasia. Arch Surg 1983;118:62-4.

36 Shemesh E, Bat L, Niv Y, et al. Melanosis coli within an adenomatous polyp. Gastrointest Endosc 1983;29:327-9.

37 Jass JR. Do all colorectal carcinomas arise in preexisting adenomas? World $\mathcal{F}$ Surg 1989;13:45-51.

38 Neugut AI, Jacobson JS, Urbanski SJ. Which colonic adenomas become malignant? Ann Intern Med 1993;119:250-2

39 O'Brien MJ, Winawer SJ, Zauber AG, et al. The national polyp study. Patient and polyp characteristics associated with high-grade dysplasia in colorectal adenomas. Gastroenterology 1990;98:371-9.

40 Koretz RL. Malignant polyps: Are they sheep in wolves' clothing? Ann Intern Med 1993;118:63-8.

41 Atkin WS, Morson BC, Cuzick J. Long-term risk of colorectal cancer after excision of rectosigmoid adenomas. $\mathrm{N} \mathrm{Engl}$ f Med 1992;326:658-62.

42 Jorgensen OD, Kronborg O, Fenger C. The Funen Adenoma Follow-Up Study. Characteristics of patients and initial adenomas in relation to severe dysplasia. Scand $\mathcal{f}$ Gastroenterol 1993;28:239-43.

43 Nusko G, Mansmann U, Altendorf-Hofmann A, et al. Differences between colorectal adenomas removed endoscopically and surgically. Multivariate analysis of patient and polyp charcteristics of the risk for high-grade dysplasia. Hepatogastroenterology 1997;44:1063-8.

44 Nusko G, Mansmann U, Partzsch U, et al. Invasive carcinoma in colorectal adenomas. Multivariate analysis of patient and adenoma characteristics. Endoscopy 1997;29: 626-31.

45 Forrester K, Almoguera C, Han K, et al. Detection of high incidence of K-ras oncogenes during human colon tumorigenesis. Nature 1987;327:298-303.

46 Vogelstein B, Fearon ER, Hamilton SR, et al. Genetic alterations during colorectal-tumor development. $N$ Engl f Med 1988;319:525-32.

47 Ahlquist DA, Thibodeau SN. Will molecular genetic markers help predict the clinical behavior of colorectal neoplasia? Gastroenterology 1992;102:1419-21.

48 Ohue $\mathrm{M}$, Tomita N, Monden T, et al. A frequent alteration of p53 gene in carcinoma in adenoma of colon. Cancer Res 1994;54:4798-804.

49 Bertram PD. Melanosis coli: a consequence of "alternative therapy" for psoriasis. Am f Gastroenterol 1993;88:971.

50 Bockus HL, Willard JH, Bank J. The etiologic significance of the anthracene laxatives: A report of forty-one cases. fAMA 1933;101:1-6.

51 Spear GS. Melanosis coli. Experimental observations on its production and elimination in twenty-three cases. $\mathrm{Am} \mathcal{F}$ Surg 1951;82:631-7. 